\title{
Effect of oil palm and jute fiber treatment on mechanical performance of epoxy hybrid composites
}

\begin{abstract}
In this work, oil palm empty fruit bunch (EFB) and jute fibers were treated with 2-hydroxy ethyl acrylate (2-HEA) to improve interfacial bonding of oil palm EFB and jute fibers with epoxy matrix. Hybrid composites were fabricated by incorporation of modified oil palm EFB and jute fibers into an epoxy matrix by the hand lay-up technique. Mechanical (flexural and impact) and morphological properties of modified hybrid composites were measured. Results indicated that flexural and impact properties of modified fiber-reinforced hybrid composites improved as compared to untreated hybrid composites due to better fiber/matrix interfacial bonding, which was confirmed by scanning electron microscopy. We confirmed that treated oil palm/jute hybrid composite may be fabricated by advanced techniques such as resin transfer molding, extrusion, and injection molding for industrial applications in the automotive sector.
\end{abstract}

Keyword: 2-hydroxy ethyl acrylate; Epoxy; Fibers; Hybrid composites; Mechanical properties 